\title{
Representations of quivers on abelian categories and monads on projective varieties
}

\author{
Marcos Jardim and Daniela Moura Prata \\ IMECC - UNICAMP \\ Departamento de Matemática \\ Caixa Postal 6065 \\ 13083-970 Campinas-SP, Brazil
}

\begin{abstract}
We consider representations of quivers in arbitrary categories and twisted representations of quivers in arbitrary tensor categories. We show that if $\mathcal{A}$ is an abelian category, then the category of representations of a quiver in $\mathcal{A}$ is also abelian, and that the category of twisted linear representations of a quiver is equivalent to the category of linear (untwisted) representations of a different quiver. We conclude by discussing how representations of quivers arise naturally in certain important problems concerning monads ans sheaves on projective varieties.
\end{abstract}

\section{Introduction}

Quivers are a valuable tool in the theory of finite dimensional associative algebras and their representations. Moreover, linear representations of quivers are a beautiful subject in itself, with many interesting connections with other areas. Recently, many authors have considered representations of quivers in arbitrary categories, see in particular [8], motivated by the relevance of such concept in algebraic geometry and mathematical physics. More precisely, representations of quivers in the category of vector bundles or coherent sheaves on a projective variety, called quiver bundles or quiver sheaves, unify many of the vector bundles with extra structure which have been previously considered in the literature (e.g. Higgs bundles, coherent systems, 
holomorphic triples, etc), see $[2,8]$. Several recent papers also consider quiver bundles in connection with string theory, see for instance $[4,16]$.

In this paper, we consider representations of quivers in arbitrary categories and twisted representations of quivers in arbitrary tensor categories, as well as some applications of these concepts to the study of certain problems concerning vector bundles over projective varieties.

First, we prove that if $\mathcal{A}$ is an additive (abelian) category, then the category of representations of a quiver in $\mathcal{A}$ is also additive (abelian), showing that the category of representations often inherits some of the properties of the original category. We also discuss how functors between categories induce functors between the respective categories of representations, and show that such induced functors also inherit properties from the original ones.

Next, we consider twisted representations of quivers in arbitrary tensor categories, and show that the category of twisted linear representations of a quiver is equivalent to the category of linear (untwisted) representations of a different quiver.

Finally, we turn to one of the original motivations behind this project by discussing the theory of monads on a projective variety $X$ from a categorical point of view, and showing that these can be regarded as representations of a quiver with relation in the category of vector bundles over $X$. We then focus on a particular class of sheaves on projective space, so-called linear sheaves, and show that the category of such sheaves is equivalent to a subcategory of the category of twisted linear representations of a quiver with relations. We look at examples in which geometric properties of sheaves are translated into algebraic properties of the corresponding twisted representations, and vice versa. This leads to a possibly (and hopefully) fruitful application of representation theoretical methods to the study of vector bundles over projective varieties.

Acknowledgments. MJ is partially supported by the CNPq grant number 305464/2007-8 and the FAPESP grant number 2005/04558-0. DMP's research is supported by the FAPESP grant number 2007/074693. We thank Vyacheslav Futorny, Adriano Moura, Flávio Coelho, Plamen Koshlukov and Eduardo Marcos for their valuable comments in various stages of this project. 


\section{Representations of Quivers}

Recall that a quiver $Q$ consists of a pair $\left(Q_{0}, Q_{1}\right)$ where $Q_{0}$ is the set of vertices and $Q_{1}$ is the set of arrows, and we have two maps $t, h: Q_{1} \rightarrow Q_{0}$ named tail and head. A path in the quiver is a sequence of arrows

$$
p=a_{1} a_{2} \cdots a_{n}
$$

such that $h\left(a_{i+1}\right)=t\left(a_{i}\right), i=1, \cdots, n-1$.

$$
\stackrel{a_{n}}{\longrightarrow} \stackrel{a_{n-1}}{\longrightarrow} \ldots \stackrel{a_{2}}{\longrightarrow} \stackrel{a_{1}}{\longrightarrow}
$$

We say that the path starts in $t\left(a_{n}\right)$ and ends in $h\left(a_{1}\right)$. The path algebra $k Q$ is the associative algebra generated by all paths of $Q$ with the product given by concatenation of paths. A relation $R$ in $Q$ is a sum of paths $p_{i} \in k Q, R=\sum_{i=1}^{n} p_{i}$, such that $t\left(p_{i}\right)=t\left(p_{j}\right)$ and $h\left(p_{i}\right)=h\left(p_{j}\right), i, j=1, \ldots, n$. Given some relations $R_{j}=\sum_{i=1}^{n_{j}} p_{i}^{j}$, $j=1, \ldots, m, p_{i}^{j} \in k Q$, the path algebra with relations is given by the quotient $k Q / I$, where $I$ is the ideal of $k Q$ generated by the relations $R_{j}, j=1, \ldots, m$.

2.1. Representations of quivers in additive and abelian categories. So let $\mathcal{A}$ be a category and $Q$ a quiver. Our first goal is to consider representations of quivers in arbitrary categories. In what follows, we will omit the usual categorical definitions like additive, abelian and tensor categories, and functors between them; we refer to the definitions found in $[7,13]$.

Definition. A representation of $Q$ in $\mathcal{A}$ consists of

- a collection of objects $\left\{V_{i}\right\}, i \in Q_{0}$;

- a collection of morphisms $\left\{\varphi_{a}\right\}, a \in Q_{1}$, where $\varphi_{a} \in \operatorname{Hom}_{\mathcal{A}}\left(V_{t(a)}\right.$, $\left.V_{h(a)}\right)$.

The representations of $Q$ in $\mathcal{A}$ is a category that we denote by $\operatorname{Rep}(Q, \mathcal{A})$.

It is interesting to observe that the category of representations $\operatorname{Rep}(Q, \mathcal{A})$ inherits some of the properties of the original category $\mathcal{A}$; in particular, one has the following result.

Theorem 1. If $\mathcal{A}$ is additive (abelian) then $\operatorname{Rep}(Q, \mathcal{A})$ is also additive (abelian). 
Proof. We prove that if $\mathcal{A}$ is additive, then $\operatorname{Rep}(Q, \mathcal{A})$ is additive. To do that we have to check that $\operatorname{Rep}(Q, \mathcal{A})$ satisfies some conditions, see [7].

(i) $\operatorname{Rep}(Q, \mathcal{A})$ has a zero object.

Since $\mathcal{A}$ is additive, we can take the object $V_{a}=0 \in$ $\operatorname{Obj}(\mathcal{A}), \forall a \in Q_{1}$, and $\varphi_{a}=0 \in \operatorname{Hom}_{\mathcal{A}}(0,0)$.

(ii) If $X, Y, Z \in \operatorname{Obj}(\mathcal{R})$ then $\operatorname{Hom}_{\mathcal{R}}(X, Y)$ is an abelian group and

$$
\operatorname{Hom}_{\mathcal{R}}(X, Y) \times \operatorname{Hom}_{\mathcal{R}}(Y, Z) \rightarrow \operatorname{Hom}_{\mathcal{R}}(X, Z)
$$

is bi-additive.

Let $X=(V, \phi), Y=(W, \psi)$ and $Z=(U, \eta)$ be objects of $\mathcal{R}=\operatorname{Rep}(Q, \mathcal{A})$. As $\operatorname{Hom}_{\mathcal{A}}\left(V_{i}, W_{i}\right)$ is an abelian group for all $i \in Q_{0}, \operatorname{Hom}_{\mathcal{R}}(X, Y)$ is also an abelian group. It follows that

$$
\operatorname{Hom}_{\mathcal{R}}(X, Y) \times \operatorname{Hom}_{\mathcal{R}}(Y, Z) \rightarrow \operatorname{Hom}_{\mathcal{R}}(X, Z)
$$

is bi-additive because for each $i \in Q_{0}$, the pairing

$$
\operatorname{Hom}_{\mathcal{A}}\left(V_{i}, W_{i}\right) \times \operatorname{Hom}_{\mathcal{A}}\left(W_{i}, U_{i}\right) \rightarrow \operatorname{Hom}_{\mathcal{A}}\left(V_{i}, U_{i}\right)
$$

is bi-additive.

(iii) $\operatorname{Rep}(Q, \mathcal{A})$ has coproducts.

Let $(V, \phi)$ and $(W, \psi)$ be objects of $\operatorname{Rep}(Q, \mathcal{A})$. As $\mathcal{A}$ is additive, for all $i \in Q_{0}, U_{i}=V_{i} \oplus W_{i}$ is an object of $\mathcal{A}$ with the maps $l_{i}: V_{i} \rightarrow U_{i}$ and $h_{i}: W_{i} \rightarrow U_{i}$.

We want to define the direct sum of $(V, \phi)$ and $(W, \psi)$. Consider the diagram

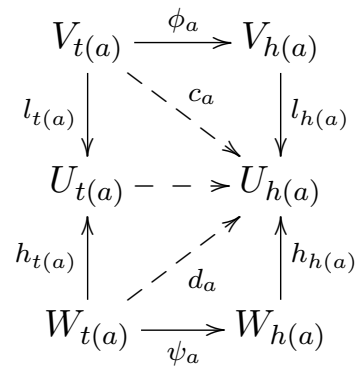

where $c_{a}=l_{h(a)} \circ \phi_{a}$ and $d_{a}=h_{h(a)} \circ \psi_{a}$.

By a property of coproducts, there exists an unique morphism

$$
\eta_{a}: U_{t(a)} \rightarrow U_{h(a)}
$$


such that $c_{a}=\eta_{a} l_{t(a)}$ and $d_{a}=\eta_{a} h_{t(a)}$. Then,

$$
l_{h(a)} \phi_{a}=\eta_{a} l_{t(a)} \text { and } h_{h(a)} \psi_{a}=\eta_{a} h_{t(a)} \text {. }
$$

Let $(U, \eta)$ be the representation $U_{i}=V_{i} \oplus W_{i}$ for $i \in Q_{0}$ and for each $a \in Q_{1}, \eta_{a}: U_{t(a)} \rightarrow U_{h(a)}$ as above. Then $(U, \eta)$ is the direct sum of $(V, \phi)$ and $(W, \psi)$ with the morphisms

$L=\left\{l_{i}\right\}_{i \in Q_{0}}:(V, \phi) \rightarrow(U, \eta)$ and $H=\left\{h_{i}\right\}_{i \in Q_{0}}:(W, \psi) \rightarrow(U, \eta)$.

Therefore $\operatorname{Rep}(Q, \mathcal{A})$ is an additive category.

If $\mathcal{A}$ is abelian, we have to verify other four conditions.

(1) Every morphism has kernel and cokernel.

Let $f:(V, \phi) \rightarrow(W, \psi)$ be a morphism. We want to define ker $f$. As $\mathcal{A}$ is abelian, for each $i \in Q_{0}, f_{i}: V_{i} \rightarrow W_{i}$ is a morphism then it has a kernel $\left(V_{i}^{\prime}, \mu_{i}\right)$

$$
\mu_{i}: V_{i}^{\prime} \rightarrow V_{i} \text {, with } f_{i} \mu_{i}=0, \forall i \in Q_{0} .
$$

For each $a \in Q_{1}$ we have $f_{h(a)}\left(\phi_{a} \mu_{t(a)}\right)=0$ then there is an unique morphism $\phi_{a}^{\prime}: V_{t(a)}^{\prime} \rightarrow V_{h(a)}^{\prime}$ such that $\phi_{a} \mu_{t(a)}=$ $\mu_{h(a)} \phi_{a}^{\prime}$.

If we set

$$
\mu=\left\{\mu_{i}\right\}_{i \in Q_{0}}:\left(V^{\prime}, \phi^{\prime}\right) \rightarrow(V, \phi)
$$

where $V^{\prime}=\left\{V_{i}^{\prime}\right\}_{i \in Q_{0}}$ and $\phi^{\prime}=\left\{\phi_{a}^{\prime}\right\}_{a \in Q_{1}}$, the diagram commutes

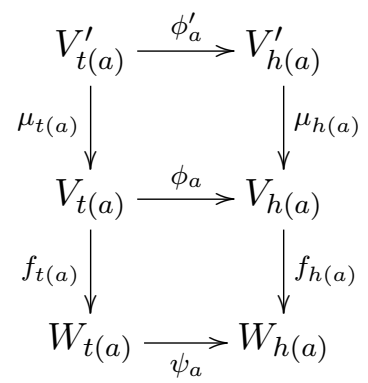

then $f \circ \mu=0$. One can see that $\operatorname{ker} f=\left(\left(V^{\prime}, \phi^{\prime}\right), \mu\right)$ is in fact kernel of $f$.

Similarly one can see that $f$ has cokernel. 
(2) Every monomorphism is the kernel of its cokernel.

Let $f:(V, \phi) \rightarrow(W, \psi)$ be a monomorphism. Then $f_{i}:$ $V_{i} \rightarrow W_{i}$ is a monomorphism for each $i \in Q_{0}$ and if $\left(W_{i}^{\prime}, c_{i}\right)=$ coker $f_{i}$ we have $\left(V_{i}, f_{i}\right)=\operatorname{ker} c_{i}, \forall i \in Q_{0}$. As $c_{h(a)} \psi_{a}$ : $W_{t(a)} \rightarrow W_{h(a)}^{\prime}$ is such that $\left(c_{h(a)} \psi_{a}\right) f_{t(a)}=0$ there is an unique $\psi_{a}^{\prime}: W_{t(a)}^{\prime} \rightarrow W_{h(a)}^{\prime}$ with $c_{h(a)} \psi_{a}=c_{t(a)} \psi_{a}^{\prime}$

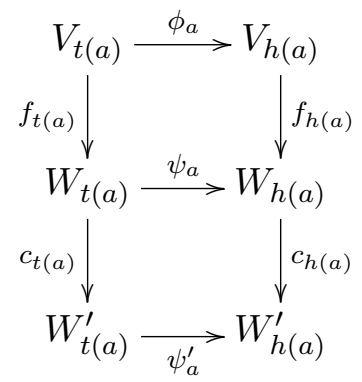

and then $\left(\left(W^{\prime}, \psi^{\prime}\right), c\right)=$ coker $f$. We have $\left(V_{i}, f_{i}\right)=\operatorname{ker} c_{i}$ and therefore $((V, \phi), f)=\operatorname{ker} c$.

(3) Every epimorphism is cokernel of its kernel.

Let $f:(V, \phi) \rightarrow(W, \psi)$ be an epimorphism. Then for each $i \in Q_{0} f_{i}: V_{i} \rightarrow W_{i}$ is an epimorphism. As $\mathcal{A}$ is abelian if $\left(V_{i}^{\prime}, k_{i}\right)=\operatorname{ker} f_{i}$ we have $f_{i} k_{i}=0, \forall i \in Q_{0}$. As $\phi_{a} k_{t(a)}$ : $V_{t(a)}^{\prime} \rightarrow V_{h(a)}$ is such that $f_{h(a)}\left(\phi_{a} k_{t(a)}\right)=0$ there is an unique $\phi_{a}^{\prime}: V_{t(a)}^{\prime} \rightarrow V_{h(a)}^{\prime}, a \in Q_{1}$, such that $\phi_{a} k_{t(a)}=k_{h(a)} \phi_{a}^{\prime}$ and $\left(\left(V^{\prime}, \phi^{\prime}\right), k\right)=\operatorname{ker} f$.

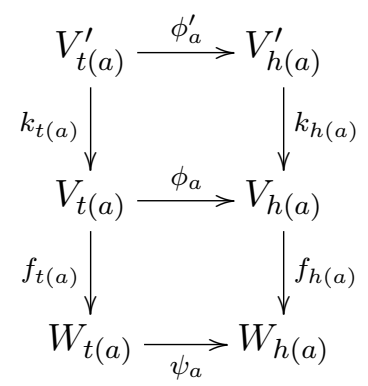

We also have $\left(W_{i}, f_{i}\right)=$ coker $k_{i}$ and $f_{i}$ is an epimorphism therefore $((W, \psi), f)=$ coker $k$ and follows the result. 
(4) Every morphism can be written as composition of epimorphism and monomorphism.

Let $f:(V, \phi) \rightarrow(W, \psi)$ be a morphism. Since $\mathcal{A}$ is abelian we know that for each $i \in Q_{0}, f_{i}: V_{i} \rightarrow W_{i}$ can be written as $f_{i}=h_{i} g_{i}$, where $g_{i}: V_{i} \rightarrow W_{i}^{\prime}$ is epimorphism and $h_{i}: W_{i}^{\prime} \rightarrow$ $W_{i}$ is monomorphism, with $W_{i}^{\prime} \in \operatorname{Obj}(\mathcal{A})$. By a lemma the diagram

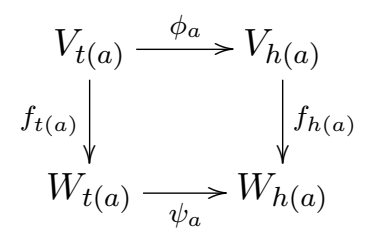

commutes, then for each $a \in Q_{1}$ there is an unique

$$
\psi_{a}^{\prime}: W_{t(a)}^{\prime} \rightarrow W_{h(a)}^{\prime}
$$

such that

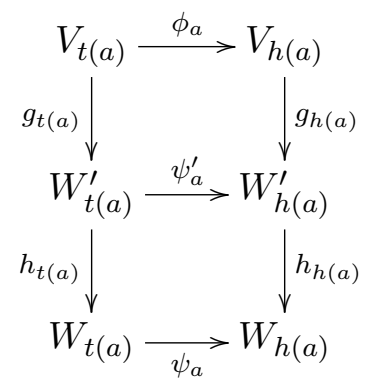

commutes, that is, $\psi_{a}^{\prime} g_{t(a)}=g_{h(a)} \phi_{a}$ and $\psi_{a} h_{t(a)}=h_{h(a)} \psi_{a}^{\prime}$. Then if we take the representation $\left(W^{\prime}, \phi^{\prime}\right)$ and the morphisms $h=\left\{h_{i}\right\}_{i \in Q_{0}}:\left(W^{\prime}, \psi^{\prime}\right) \rightarrow(W, \psi)$ and $g:\left\{g_{i}\right\}_{i \in Q_{0}}(V, \phi) \rightarrow$ $(W, \psi)$ follows that $g$ is epimorphism, $h$ is monomorphism and $f=h \circ g$.

Remark 2. We believe that other standard categorical properties of $\mathcal{A}$, like the existence of sufficiently many projectives and injectives, will also be inherited by the category of representations $\operatorname{Rep}(Q, \mathcal{A})$. 
If $\mathcal{A}$ is an additive category, one can also consider representations of quivers with relations in $\mathcal{A}$.

Definition. Let $\mathcal{A}$ be an additive category, $Q$ a quiver and $R=$ $\left\{R_{1}, \ldots\right.$,

$\left.R_{m}\right\}$ be a set of relations in $Q$, where $R_{i}=\sum_{j=1}^{n_{i}} p_{j}^{i}, p_{j}^{i}=a_{j 1}^{i} \cdots a_{j l_{j}}^{i}$. $A$ representation $(V, \phi)$ in $\mathcal{A}$ satisfies the relations if $\sum_{j=1}^{n_{i}} \phi_{p_{j}^{i}}=0$ for each $i=1, \cdots, m$, where $\phi_{p_{j}^{i}}=\phi_{a_{j 1}^{i}} \cdots \phi_{a_{j l}^{i}}$.

We denote by $\operatorname{Rep}((Q, R), \mathcal{A})$ the category of representations of the quiver with relations. It is not difficult to see that $\operatorname{Rep}((Q, R), \mathcal{A})$ is a full additive subcategory of $\operatorname{Rep}(Q, \mathcal{A})$.

2.2. Induced functors. Let $\mathcal{A}$ and $\mathcal{B}$ be categories, $Q$ a quiver and $\mathbf{F}: \mathcal{A} \rightarrow \mathcal{B}$ a functor. We can define a functor $\mathbf{F}_{Q}: \operatorname{Rep}(Q, \mathcal{A}) \rightarrow$ $\operatorname{Rep}(Q, \mathcal{B})$ in the following way:

- given an object $(V, \phi) \in \operatorname{Rep}(Q, \mathcal{A}), \mathbf{F}_{Q}((V, \phi))=(W, \psi)$ where $W_{i}=\mathbf{F}\left(V_{i}\right) \in \operatorname{Obj}(\mathcal{B}), i \in Q_{0}$, and $\psi_{a}=\mathbf{F}\left(\phi_{a}\right) \in$ $\operatorname{Hom}_{\mathcal{B}}\left(W_{t(a)}, W_{h(a)}\right), a \in Q_{1}$

- given a morphism $f:(V, \phi) \rightarrow(W, \psi), f=\left\{f_{i}\right\}, f_{i} \in$ $\operatorname{Hom}_{\mathcal{A}}\left(V_{i}, W_{i}\right)$ we define $\mathbf{F}_{Q}(f)=g$, where $g_{i}=\mathbf{F}\left(f_{i}\right) \in$ $\operatorname{Hom}_{\mathcal{B}}\left(\mathbf{F}\left(V_{i}\right), \mathbf{F}\left(W_{i}\right)\right), i \in Q_{0}$.

It turns out that the induced functor $\mathbf{F}_{Q}$ also inherits properties of the functor original $\mathbf{F}$, as in the following Proposition.

Proposition 3. Let $\mathbf{F}: \mathcal{A} \rightarrow \mathcal{B}$ be a functor.

- If $\mathbf{F}$ is an equivalence of categories, then so is the induced functor $\mathbf{F}_{Q}$ for any quiver $Q$;

- If $\mathcal{A}$ and $\mathcal{B}$ are abelian categories and $\mathbf{F}$ is an exact functor, then so is the induced functor $\mathbf{F}_{Q}$ for any quiver $Q$.

Proof. First, suppose $\mathbf{F}$ is an equivalence of categories. To show that $\mathbf{F}_{Q}$ is equivalence of categories we must see that $\mathbf{F}_{Q}$ is fully faithful and essentially surjective.

Let $X=(V, \phi)$ and $Y=(W, \psi)$ be objects of $\operatorname{Rep}(Q, \mathcal{A})$, and take $f, g \in \operatorname{Hom}(X, Y)$ such that $\mathbf{F}_{Q}(f)=\mathbf{F}_{Q}(g)$, with

$$
\mathbf{F}_{Q}: \operatorname{Hom}(X, Y) \rightarrow \operatorname{Hom}(\mathbf{F}(X), \mathbf{F}(Y)) .
$$


Then $\mathbf{F}\left(f_{i}\right)=\mathbf{F}\left(g_{i}\right), \forall i \in Q_{0}, f_{i}, g_{i} \in \operatorname{Hom}\left(V_{i}, W_{i}\right)$. Since $\mathbf{F}$ is faithful, $f_{i}=g_{i}, \forall i \in Q_{0}$ then $f=g$ therefore $\mathbf{F}_{Q}$ is also faithful.

If $\bar{g} \in \operatorname{Hom}\left(F_{Q}(X), F_{Q}(Y)\right)$ where $\bar{g}=\left\{\bar{g}_{i}\right\}_{i \in Q_{0}}, \quad \bar{g}_{i} \in$ $\operatorname{Hom}\left(F\left(V_{i}\right), F\left(W_{i}\right)\right)$, since $\mathbf{F}$ is full, for each $i \in Q_{0}$ there is a morphism $g_{i} \in \operatorname{Hom}\left(V_{i}, W_{i}\right)$ such that $\mathbf{F}\left(g_{i}\right)=\overline{g_{i}}$. Thus $\mathbf{F}(g)=\bar{g}$ where $g=\left\{g_{i}\right\}_{i \in Q_{0}}$, hence $\mathbf{F}_{Q}$ is full. We have shown that if $\mathbf{F}$ is fully faithful, then so is $\mathbf{F}_{Q}$.

Let $Y=(W, \psi) \in \operatorname{Obj}(\operatorname{Rep}(Q, \mathcal{B}))$. Since $\mathbf{F}$ is essentially surjective, there is an isomorphism $\lambda_{i}: W_{i} \rightarrow \mathbf{F}\left(V_{i}\right), \forall i \in Q_{0}, V_{i} \in$ $\operatorname{Obj}(\mathcal{A})$. For each $a \in Q_{1}$ consider the map $\phi_{a}^{\prime}=\lambda_{h(a)} \psi_{a} \lambda_{t(a)}^{-1}$. Then $\phi_{a}^{\prime} \in \operatorname{Hom}\left(\mathbf{F}\left(V_{t(a)}\right), \mathbf{F}\left(V_{h(a)}\right)\right)$ and since $\mathbf{F}$ is full, there is a map $\phi_{a} \in \operatorname{Hom}\left(V_{t(a)}, V_{h(a)}\right)$ such that $\mathbf{F}\left(\phi_{a}\right)=\phi_{a}^{\prime}$. Let $X=(V, \phi)$ where $V=\left\{V_{i}\right\}_{i \in Q_{0}}$ and $\phi=\left\{\phi_{a}\right\}_{a \in Q_{1}}$. Then $X \in \operatorname{Obj}(\operatorname{Rep}(Q, \mathcal{A}))$ and $\mathbf{F}(X) \simeq Y$ with isomorphism $\lambda$ and so $\mathbf{F}_{Q}$ is essentially surjective, therefore $\mathbf{F}_{Q}$ is equivalence of categories.

Now suppose that $\mathbf{F}$ is an exact functor and let $X=(V, \phi), Y=$ $(W, \psi), Z=(U, \lambda)$ be objects of $\operatorname{Rep}(Q, \mathcal{A}), f \in \operatorname{Hom}(X, Y)$ and $g \in \operatorname{Hom}(Y, Z)$ such that

$$
0 \longrightarrow X \stackrel{f}{\longrightarrow} Y \stackrel{g}{\longrightarrow} Z \longrightarrow 0
$$

is a short exact sequence. Then, for each $a \in Q_{1}$, the following diagram is commutative:

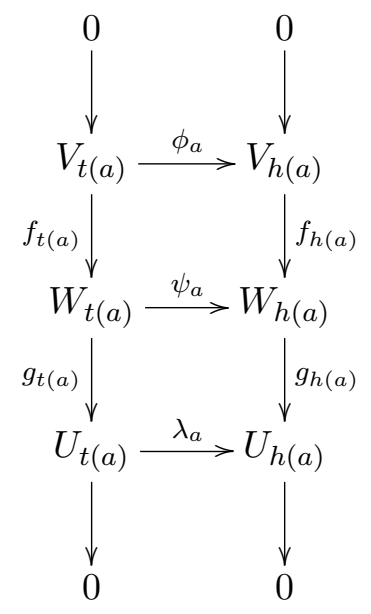


Since $\mathbf{F}$ is an exact functor, the diagram

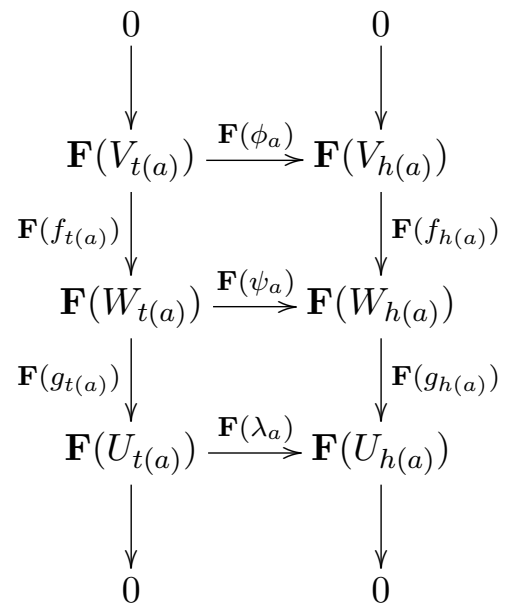

is also commutative, then

$$
0 \longrightarrow \mathbf{F}_{Q}(X) \stackrel{\mathbf{F}_{Q}(f)}{\longrightarrow} \mathbf{F}_{Q}(Y) \stackrel{\mathbf{F}_{Q}(g)}{\longrightarrow} \mathbf{F}_{Q}(Z) \longrightarrow 0
$$

is a short exact sequence and therefore $\mathbf{F}_{Q}$ is an exact functor.

\section{Twisted linear representations of quivers}

The concept of twisted representations of quivers was first introduced by Gothen and King [8, p. 88], motivated by certain problems involving vector bundles with extra structures, like Higgs bundles and holomorphic triples. Here, we will define twisted representations in an arbitrary tensor category, and, for representations in the category of vector spaces over a field, we will relate the category of twisted representations of $Q$ with the category of representations of a different quiver $\tilde{Q}$.

\subsection{Twisted representations of quivers.}

Definition. Let $Q$ be a quiver and $\mathcal{A}$ a tensor category. Fix a collection $M=\left\{M_{a}\right\}_{a \in Q_{1}}$ of objects of $\mathcal{A}$. A right $M$-twisted representation of $Q$ consists of

- a collection of objects $\left\{V_{i} \mid i \in Q_{0}\right\}$;

- a collection of morphisms $\left\{\varphi_{a}: V_{t(a)} \rightarrow V_{h(a)} \otimes M_{a} \mid a \in Q_{1}\right\}$. 
We also denote the representation by $(V, \phi)$. Alternatively, one could also consider the morphisms $\left\{\varphi_{a}: M_{a} \otimes V_{t(a)} \rightarrow V_{h(a)} \mid a \in Q_{1}\right\}$, leading to the notion of a left $M$-twisted representation of $Q$.

A morphism between two twisted representations $(V, \phi)$ and $(W, \psi)$ in $\mathcal{A}$, is a collection of morphisms $f_{i}: V_{i} \rightarrow W_{i}, i \in Q_{0}$, such that the following diagram commutes for each $a \in Q_{1}$.

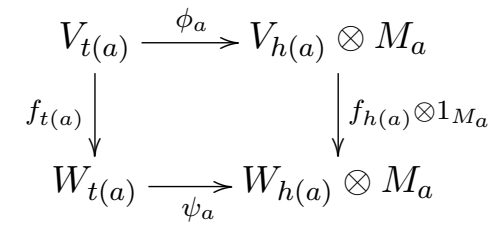

that is,

$$
\left(f_{h(a)} \otimes 1_{M_{a}}\right) \circ \phi_{a}=\psi_{a} \circ f_{t(a)}, \forall a \in Q_{1} .
$$

We have then a new category of right $M$-twisted representations of $Q$, denoted by $\operatorname{Rep}_{M}(Q, \mathcal{A})$.

Suppose $Q$ has relations $R=\left\{R_{1}, \cdots, R_{m}\right\}$, where $R_{i}=\sum_{j=1}^{n_{i}} p_{j}^{i}$, and $p_{j}^{i}=a_{j 1}^{i} \cdots a_{j l_{j}}^{i}$. Let $M=\left\{M_{a}\right\}, a \in Q_{1}$, be a collection of objects of $\mathcal{A}, M_{p_{j}^{i}}=M_{a_{j 1}^{i}} \otimes M_{a_{j 2}^{i}} \otimes \ldots \otimes M_{a_{j l_{j}}}, j=1, \ldots n_{i}, i=1, \ldots, m$ and $\tilde{M}=\bigotimes_{a \in Q_{1}} M_{a}$. Given a twisted representation $(V, \phi)$ we have induced maps

where

$$
\tilde{\phi}_{p_{j}^{i}}: V_{t\left(p_{j}^{i}\right)} \rightarrow V_{h\left(p_{j}^{i}\right)} \otimes M_{p_{j}^{i}}
$$

$$
\begin{gathered}
\tilde{\phi}_{p_{j}^{i}}=\left(\phi_{a_{j 1}^{i}} \otimes 1_{M_{a_{j 2}^{i}}} \otimes \ldots \otimes 1_{M_{a_{j l_{j}}}}\right) \circ\left(\phi_{a_{j 2}^{i}} \otimes 1_{M_{a_{j 3}^{i}}} \otimes \ldots \otimes 1_{M_{a_{j l_{j}}}}\right) \circ \ldots \\
\ldots \otimes\left(\phi_{a_{j l_{j}-1}^{i}} \otimes 1_{M_{a_{j l_{j}}^{i}}}\right) \circ \phi_{a_{j l_{j}}^{i}} .
\end{gathered}
$$

If $f_{p_{j}^{i}}: M_{p_{j}^{i}} \rightarrow \tilde{M}$ is the inclusion map and $\phi_{p_{j}^{i}}=\left(1_{V_{h\left(p_{j}^{i}\right)}} \otimes f_{p_{j}^{i}}\right) \circ \tilde{\phi}_{p_{j}^{i}}$, we have

$$
\phi_{p_{j}^{i}}: V_{t\left(p_{j}^{i}\right)} \rightarrow V_{h\left(p_{j}^{i}\right)} \otimes \tilde{M}
$$

for $j=1, \ldots, n_{i}$ 
A twisted representation $(V, \phi)$ satisfies the relations if $\phi_{p_{1}^{i}}+\ldots+$ $\phi_{p_{n_{i}}^{i}}=0$, for $i=1, \cdots, m$. We have again a new category, the category of $M$-twisted representations of the quiver $Q$ with relations.

3.2. Twisted representations of quivers. Let $Q$ be a quiver, $\mathcal{A}$ be the category of finite dimensional vector spaces over a field $k$ and let $M=\left\{M_{a}\right\}, a \in Q_{1}$, be a collection of objects of $\mathcal{A}$. The main result of this section is to relate, as we mentioned before, the categories of twisted $k$-linear representations of a quiver with a category of $k$-linear representations of another quiver.

Theorem 4. The category of $M$-twisted $k$-linear representations of $Q$ is equivalent to the category of $k$-linear representations of $\tilde{Q}$, where $\tilde{Q}$ is obtained from $Q$ in the following way;

- the set of vertices is the same, that is, $Q_{0}=\tilde{Q}_{0}$;

- for each arrow $a \in Q_{1}, \tilde{Q}_{1}$ possesses $m=\operatorname{dim} M_{a}$ arrows, $a_{1}, \cdots, a_{m}$, such that $\tilde{t}\left(a_{j}\right)=t(a)$ and $\tilde{h}\left(a_{j}\right)=h(a)$, where $\tilde{t}, \tilde{h}$ are the tail and head maps of $\tilde{Q}$.

Proof. Suppose that $Q$ is $Q_{0}=(1,2)$ and $Q_{1}=(a)$ with $t(a)=1$ and $h(a)=2$

$$
1 \stackrel{a}{\longrightarrow} 2 .
$$

Fix $M_{a}$ a $k$-vector space, $\operatorname{dim} M_{a}=m$. We show that $\mathcal{C}=$ $\operatorname{Rep}_{M}(Q)$ is equivalent to $\mathcal{D}=\operatorname{Rep}(\tilde{Q})$, where $\tilde{Q}=\left(\tilde{Q}_{0}, \tilde{Q}_{1}\right)$ with $\tilde{Q}_{0}=Q_{0}$ and $\tilde{Q}_{1}=\left(a_{1}, \ldots, a_{m}\right), t\left(a_{i}\right)=t(a)$ and $h\left(a_{i}\right)=h(a)$, for $i=1, \ldots, m$.

We construct a functor that is an equivalence of categories. Let $\{V, W\}$,

$\left\{\phi: V \rightarrow W \otimes M_{a}\right\}$ be a representation of $Q$. Since $L\left(V, W \otimes M_{a}\right) \simeq$ $L(V, W) \otimes M_{a}$, if $\left\{h_{1}, \ldots, h_{m}\right\}$ is a basis for $M_{a}$ and $\phi \in L\left(V, W \otimes M_{a}\right)$, there are $\phi_{1}, \cdots, \phi_{m} \in L(V, W)$ such that

$$
\phi=\sum_{i=1}^{m} \phi_{i} \otimes h_{i} .
$$


Fix an order in the set of arrows between two vertices of the quiver $\tilde{Q}$ and consider the functor

$$
\mathbf{F}: \operatorname{Rep}_{M}(Q) \rightarrow \operatorname{Rep}(\tilde{Q})
$$

such that

- for each object $X=\left(\left\{V_{t(a)}, V_{h(a)}\right\}, \phi\right) \in \operatorname{Rep}_{M}(Q)$

$$
\mathbf{F}(X)=\mathbf{F}\left(\left\{V_{t(a)}, V_{h(a)}\right\}, \phi\right)=\left(\left\{V_{t(a)}, V_{t(a)}\right\}, \tilde{\phi}\right)
$$

where $\tilde{\phi}=\left\{\phi_{1}, \ldots, \phi_{m}\right\}$ are obtained as in (1) and $\phi_{n}$ is associated to the $n$-th arrow of $\tilde{Q}$;

- for each morphism $f=\left\{f_{t(a)}, f_{h(a)}\right\}$ between $X=\left(\left\{V_{t(a)}, V_{h(a)}\right\}\right.$, $\phi)$ and $Y=\left(\left\{W_{t(a)}, W_{h(a)}\right\}, \psi\right)$

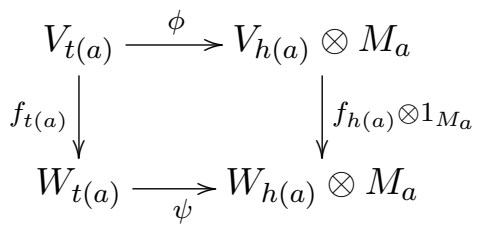

we define $\mathbf{F}(f)=f$.

It's easy to see that $f=\left\{f_{t(a)}, f_{h(a)}\right\}$ is a morphism between the representations $\left(\left\{V_{t(a)}, V_{h(a)}\right\},\left\{\phi_{1}, \ldots, \phi_{m}\right\}\right)$ and $\left(\left\{W_{t(a)}, W_{h(a)}\right\}\right.$, $\left.\left\{\psi_{1}, \ldots \psi_{m}\right\}\right)$, and that $\mathbf{F}$ is a functor. Now we must show that $\mathbf{F}$ is an equivalence of categories. To see this is sufficient to show that $\mathbf{F}$ is fully faithful functor and essentially surjective, see [7, p. 71].

(1) $\mathbf{F}: \operatorname{Hom}_{\mathcal{C}}(X, Y) \rightarrow \operatorname{Hom}_{\mathcal{D}}(\mathbf{F}(X), \mathbf{F}(Y))$ is injective and surjective. Clearly $\mathbf{F}$ is injective. Let $g \in \operatorname{Hom}_{\mathcal{D}}(\mathbf{F}(X), \mathbf{F}(Y))$, $g=\left\{g_{i}\right\}_{i \in Q_{0}}$ such that

$$
\psi_{i} \circ g_{t(a)}=g_{h(a)} \circ \phi_{i}, i=1, \ldots, m .
$$

Then $f=\left\{g_{i}\right\}_{i \in Q_{0}} \in \operatorname{Hom}_{\mathcal{C}}(X, Y)$ is such that $\mathbf{F}(f)=g$. In fact

$$
\begin{aligned}
& \psi g_{t(a)}=\left(\sum_{j=1}^{m} \psi_{j} \otimes h_{j}\right) g_{t(a)}=\sum_{j=1}^{m}\left(\left(\psi_{j} \otimes h_{j}\right) g_{t(a)}\right)=\sum_{j=1}^{m} \psi_{j} g_{t(a)} \otimes h_{j}= \\
& \sum_{j=1}^{m} g_{h(a)} \phi_{j} \otimes h_{j}=\sum_{j=1}^{m} g_{h(a)} \phi_{j} \otimes 1_{M_{a}} h_{j}=\sum_{j=1}^{m}\left(g_{h(a)} \otimes 1_{M_{a}}\right)\left(\phi_{j} \otimes h_{j}\right)=
\end{aligned}
$$




$$
\left(g_{h(a)} \otimes 1_{M_{a}}\right)\left(\sum_{j=1}^{m} \phi_{j} \otimes h_{j}\right)=\left(g_{h(a)} \otimes 1_{M_{a}}\right) \phi .
$$

Therefore $\mathbf{F}$ is fully faithful.

(2) Let $\bar{Y} \in \operatorname{Obj}(\mathcal{D}), \bar{Y}=(V, \bar{\phi})$ where $V=\left\{V_{t(a)}, V_{h(a)}\right\}$ and $\bar{\phi}=\left\{\phi_{1}, \ldots, \phi_{m}\right\}$ with $\phi_{j}: V_{t(a)} \rightarrow V_{h(a)}, j=1, \ldots, m$. Let $X=(V, \phi)$ with

$$
\phi=\sum_{j=1}^{m} \phi_{j} \otimes h_{j} .
$$

Then $\bar{Y}=\mathbf{F}(X)$. Therefore $\mathbf{F}: \mathcal{C} \rightarrow \mathcal{D}$ is an equivalence of categories. Since the construction of the proof depends on the arrow, the proof for the general quiver follows from performing this construction for each arrow.

Remark 5. The particular functor constructed in the proof of the theorem depend on two choices: the choice of an order in the set of arrows between two vertices of the quiver $\tilde{Q}$, and the choice of bases for the vector spaces $M_{a}$ for each $a \in Q_{1}$. It is reasonable to expect that different choices lead to naturally isomorphic functors, although the authors have not been able to establish this claim.

Proposition 6. Let $M=\left\{M_{a}\right\}_{a \in Q_{1}}$ be a collection of finite dimensional $k$-vector spaces. Given $M^{\prime}=\left\{M_{a}^{\prime}\right\}_{a \in Q_{1}}$ a collection of vector subspaces, $M_{a}^{\prime} \subset M_{a}, a \in Q_{1}$, there is a fully faithful functor $\mathbf{F}: \operatorname{Rep}_{M^{\prime}}(Q) \rightarrow \operatorname{Rep}_{M}(Q)$.

Proof. Let $\mathcal{C}=\operatorname{Rep}_{M^{\prime}}(Q)$ and $\mathcal{D}=\operatorname{Rep}_{M}(Q)$. Let $X=(V, \phi) \in$ $\operatorname{Obj}(\mathcal{C})$ then $\phi_{a}: V_{t(a)} \rightarrow V_{h(a)} \otimes M_{a}^{\prime}$ for each $a \in Q_{1}$. Let $\varepsilon_{a}: M_{a}^{\prime} \rightarrow$ $M_{a}$ be the inclusion map. Consider the map

$$
\bar{\phi}_{a}: V_{t(a)} \rightarrow V_{h(a)} \otimes M_{a}
$$

given by $\bar{\phi}_{a}=\left(1_{V_{h(a)}} \otimes \varepsilon_{a}\right) \circ \phi_{a}$ for each $a \in Q_{1}$. We define the functor $\mathbf{F}: \mathcal{C} \rightarrow \mathcal{D}$

- for $X=(V, \phi) \in \operatorname{Obj}(\mathcal{C})$ we have

$$
\mathbf{F}(X)=(V, \bar{\phi})
$$


where $\bar{\phi}_{a}=\left(1_{V_{h(a)}} \otimes \varepsilon_{a}\right) \circ \phi_{a}, a \in Q_{1}$;

- if $f: X \rightarrow Y$ is a morphism and $X=(V, \phi), Y=(W, \psi)$ are objects of $\mathcal{C}$ then $\mathbf{F}(f)=f$ is a morphism between $\mathbf{F}(V, \phi)$ and $\mathbf{F}(W, \psi)$.

In fact we must show that the diagram bellow is commutative

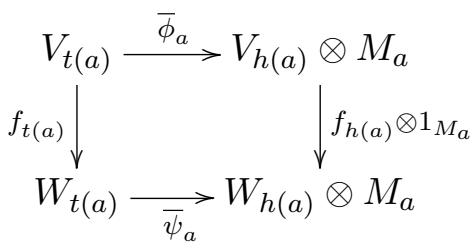

We have

and

$$
\begin{gathered}
\bar{\psi}_{a} f_{t(a)}=\left(\left(1_{W_{h(a)}} \otimes \varepsilon_{a}\right) \psi_{a}\right) f_{t(a)}=\left(1_{W_{h(a)}} \otimes \varepsilon_{a}\right)\left(\psi_{a} f_{t(a)}\right) \\
=\left(1_{W_{h(a)}} \otimes \varepsilon_{a}\right)\left(f_{h(a)} \otimes 1_{M_{a}^{\prime}}\right) \phi_{a}
\end{gathered}
$$

$$
\left(f_{h(a)} \otimes 1_{M_{a}}\right) \bar{\phi}_{a}=\left(f_{h(a)} \otimes 1_{M_{a}}\right)\left(1_{V_{h(a)}} \otimes \varepsilon_{a}\right) \phi_{a} .
$$

Since $1_{W_{h(a)}} f_{h(a)}=f_{h(a)} 1_{V_{h(a)}}$ and $\varepsilon_{a} 1_{M_{a}^{\prime}}=1_{M_{a}} \varepsilon_{a}$ note that

$$
\left(1_{W_{h(a)}} \otimes \varepsilon_{a}\right)\left(f_{h(a)} \otimes 1_{M_{a}^{\prime}}\right)=\left(f_{h(a)} \otimes 1_{M_{a}}\right)\left(1_{V_{h(a)}} \otimes \varepsilon_{a}\right)
$$

then

$$
\bar{\psi}_{a} f_{t(a)}=\left(f_{h(a)} \otimes 1_{M_{a}}\right) \bar{\phi}_{a}
$$

and $\mathbf{F}(f) \in \operatorname{Hom}_{\mathcal{D}}(\mathbf{F}(X), \mathbf{F}(Y))$.

If $g: Y \rightarrow Z$ is a morphism where $Z=(U, \eta)$ then is easy to see that $g f: X \rightarrow Z$ is well defined and that $\mathbf{F}(f g)=\mathbf{F}(f) \mathbf{F}(g)$ e therefore $\mathbf{F}$ is a functor.

Now we have to show that $\mathbf{F}$ is fully faithful, that is, for each object $X, Y \in \operatorname{Obj}(\mathcal{C})$

$$
\mathbf{F}: \operatorname{Hom}_{\mathcal{C}}(X, Y) \rightarrow \operatorname{Hom}_{\mathcal{D}}(\mathbf{F}(X), \mathbf{F}(Y))
$$

is surjective and injective. 
Let $X=(V, \phi)$ and $Y=(W, \psi)$ be objects of $\operatorname{Rep}_{M^{\prime}}(Q)$ and let $g=\left\{g_{i}\right\}_{i \in Q_{0}} \in \operatorname{Hom}_{\mathcal{D}}(\mathbf{F}(X), \mathbf{F}(Y))$. Then the diagram commutes

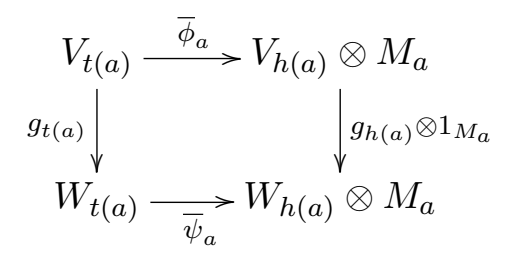

that is,

$$
\bar{\psi}_{a} g_{t(a)}=\left(g_{h(a)} \otimes 1_{M_{a}}\right) \bar{\phi}_{a}, \forall a \in Q_{1}
$$

then

$$
\left(\left(1_{W_{h(a)}} \otimes \varepsilon_{a}\right) \psi_{a}\right) g_{t(a)}=\left(g_{h(a)} \otimes 1_{M_{a}}\right)\left(\left(1_{V_{h(a)}} \otimes \varepsilon_{a}\right) \phi_{a}\right)
$$

and so

$$
\left(1_{W_{h(a)}} \otimes \varepsilon_{a}\right)\left(\psi_{a} g_{t(a)}\right)=\left(g_{h(a)} \otimes 1_{M_{a}}\right)\left(1_{V_{h(a)}} \otimes \varepsilon_{a}\right) \phi_{a} .
$$

Note that

$$
\left(g_{h(a)} \otimes 1_{M_{a}}\right)\left(1_{V_{h(a)}} \otimes \varepsilon_{a}\right)=\left(1_{W_{h(a)}} \otimes \varepsilon_{a}\right)\left(g_{h(a)} \otimes 1_{M_{a}^{\prime}}\right)
$$

then

$$
\left(1_{W_{h(a)}} \otimes \varepsilon_{a}\right)\left(\psi_{a} g_{t(a)}\right)=\left(1_{W_{h(a)}} \otimes \varepsilon_{a}\right)\left(\left(g_{h(a)} \otimes 1_{M_{a}^{\prime}}\right) \phi_{a}\right) .
$$

We have that $f=\left\{g_{i}\right\}_{i \in Q_{1}} \in \operatorname{Hom}_{\mathcal{C}}(X, Y)$ is such that $\mathbf{F}(f)=g$ then $\mathbf{F}: \operatorname{Hom}_{\mathcal{C}}(X, Y) \rightarrow \operatorname{Hom}_{\mathcal{D}}(\mathbf{F}(X), \mathbf{F}(Y))$ is surjective. Clearly $\mathbf{F}$ is injective therefore is fully faithful.

In the previous Proposition one may choose each $M_{a}^{\prime}$ to be a 1dimensional subspace of $M_{a}$; together with Theorem 4 , we have the following statement.

Corollary 7. $\operatorname{Rep}(Q)$ is equivalent to a full subcategory of $\operatorname{Rep}_{M}(Q)$. 


\section{Monads on projective varieties and representations of quivers}

Representations of quivers arise naturally in certain important problems concerning vector bundles on projective varieties. In this Section, we will discuss two such examples: first, we observe that monads over a projective variety $X$ can be regarded as representations of quivers in the category of vector bundles over $X$; we then specialize to linear monads and linear bundles, and show that these form a category which is equivalent to a certain subcategory of a category of twisted linear representations.

We must first recall the following definition of an exact subcategory of an abelian category.

Definition. Let $\mathcal{A}$ be an abelian category. A full, additive subcategory $\mathcal{E}$ of $\mathcal{A}$ is said to be exact if the following two conditions hold:

(1) $\mathcal{E}$ is closed under extensions, i.e. if $Y$ and $Z$ are objects of $\mathcal{E}$, then any extension of $Z$ by $Y$ is also objects of $\mathcal{E}$;

(2) $\mathcal{E}$ is closed under direct summands, i.e. if $X$ is an object of $\mathcal{E}$ and $X \simeq Y \oplus Z$, then $Y$ and $Z$ are also objects of $\mathcal{E}$.

In what follows, we will be particularly interested in the following quiver with relation

$$
Q=\{\bullet \stackrel{a}{\longrightarrow} \bullet \stackrel{b}{\longrightarrow} \bullet\} R=\{b a\},
$$

which we denote $\left(A_{3}, b a\right)$. If $X$ is a projective variety, i.e. a projective scheme over an algebraically closed field $\mathbb{F}$ together with a given very ample invertible sheaf denoted by $\mathcal{O}_{X}(1)$, we set $\mathcal{M}(X):=$ $\operatorname{Rep}\left(\left(A_{3}, b a\right), \operatorname{Coh}(X)\right)$, where $\operatorname{Coh}(X)$ is the category of coherent sheaves of $\mathcal{O}_{X}$-modules on $X$. Let $\mathcal{V}(X)$ be the category of locally-free sheaves on $X$.

Furthermore, For any coherent sheaf $E$ on $X$, we set $E(k):=$ $E \otimes \mathcal{O}_{X}(k)$ and $H_{*}^{p}(E):=\oplus_{k \in \mathbb{Z}} H^{p}(E(k))$. Finally, $\omega_{X}$ denotes the dualizing sheaf on $X$.

4.1. Monads on projective varieties. We start by recalling the notion of a Horrocks monad, cf. [1, Definition 2.2] and [14, page 239].

Definition. A monad on $X$ is a complex of locally free sheaves

$$
\text { M. : } M_{0} \stackrel{\alpha}{\longrightarrow} M_{1} \stackrel{\beta}{\longrightarrow} M_{2}
$$


such that $\beta$ is surjective, $\alpha$ is injective. A monad is said to be Horrocks if in addition

(i) $M_{0}=\oplus_{i=1}^{r} \omega_{X}\left(k_{i}\right)$ for $k_{i} \in \mathbb{Z}$;

(ii) $M_{2}=\oplus_{j=1}^{s} \mathcal{O}_{X}\left(l_{j}\right)$ for $l_{j} \in \mathbb{Z}$;

(iii) $H_{*}^{1}\left(M_{1}\right)=H_{*}^{n-1}\left(M_{1}\right)=0$.

Remark 8. Notice that the above definition is weaker than $[12$, Definition 2.1], where the maps $\beta$ and $\alpha$ are assumed to be locally rightinvertible and locally left-invertible, respectively. In this case, the cohomology sheaf $E=\operatorname{ker} \beta / \operatorname{im} \alpha$ is only a coherent sheaf (not necessarily locally-free).

A morphism between two monads is simply a morphism of complexes. With these definitions, note that Horrocks monads on a projective scheme $X$ form a category, denoted $\mathcal{H}(X)$. It is easy to see that $\mathcal{H}(X)$ is a full, additive subcategory of $\mathcal{M}(X)$, but more is true when one restricts the class of schemes under consideration.

Definition. A projective variety $X \hookrightarrow \mathbb{P}^{n}$ of pure dimension $d$ is arithmetically Cohen-Macaulay (ACM) if its homogeneous coordinate ring $S(X)$ is a Cohen-Macaulay ring.

This is equivalent to saying that $H_{*}^{1}\left(\mathbb{P}^{n}, \mathcal{I}_{X}\right)=0$ (where $\mathcal{I}_{X}$ is the saturated ideal of $X$ ) and $H_{*}^{p}\left(\mathcal{O}_{X}\right)=0$ for every $1 \leq p \leq d-1$ [5]. In particular, $S(X)=H_{*}^{0}\left(\mathcal{O}_{X}\right)$. For instance, every complete intersection scheme $X \subset \mathbb{P}^{n}$ is ACM. Note that if $X$ is ACM, then $H_{*}^{p}\left(\omega_{X}\right)=0$ for $1 \leq p \leq d-1$, by Serre duality. Note also that $H_{*}^{p}(E)$ is a graded $S(X)$-module.

We then have the following proposition.

Proposition 9. The category $\mathcal{H}(X)$ of Horrocks' monads on a nonsingular ACM projective scheme $X$ is an exact subcategory of $\mathcal{M}(X)$.

Proof. Consider the following two objects of $\mathcal{M}(X)$

$$
\text { M• : } M_{0} \stackrel{\alpha_{M}}{\longrightarrow} M_{1} \stackrel{\beta_{M}}{\longrightarrow} M_{2} \text { andN } \bullet: N_{0} \stackrel{\alpha_{N}}{\longrightarrow} N_{1} \stackrel{\beta_{N}}{\longrightarrow} N_{2}
$$

The direct sum $\mathrm{M}_{\bullet} \oplus \mathrm{N}_{\bullet}$ is the complex

$$
M_{0} \oplus N_{0} \stackrel{\alpha_{M} \oplus \alpha_{N}}{\longrightarrow} M_{1} \oplus N_{1} \stackrel{\beta_{M} \oplus \beta_{N}}{\longrightarrow} M_{2} \oplus N_{2} .
$$


It is easy to see from the definition that if $\mathrm{M}_{\bullet} \oplus \mathrm{N}_{\bullet}$ is Horrocks, then $\mathrm{M}_{\bullet}$ and $\mathrm{N}_{\bullet}$ must also be Horrocks; in other words, $\mathcal{H}(X)$ is closed under direct summands.

To see that $\mathcal{H}(X)$ is closed under extensions, assume that $\mathrm{M}_{\bullet}$ and $\mathrm{N}_{\bullet}$ as above are two Horrocks monads. An extention of $\mathrm{M}_{\bullet}$ by $\mathrm{N}_{\bullet}$ is an object

$$
\text { L• : } L_{0} \stackrel{\alpha_{L}}{\longrightarrow} L_{1} \stackrel{\beta_{L}}{\longrightarrow} L_{2}
$$

of $\mathcal{M}(X)$ such that the following diagram is commutative

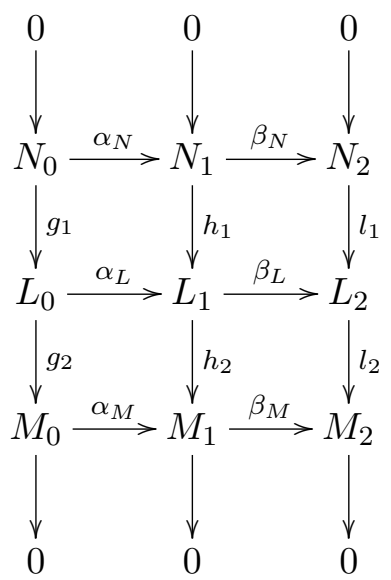

in which the columns are exact. Chasing diagrams, one easily shows that $\alpha_{L}$ is injective, and $\beta_{L}$ is surjective.

Notice also that $L_{k}$ is an extension of $M_{k}$ by $N_{k}$. Since $X$ is an ACM scheme, it follows that the first and last columns split as exact sequences, giving maps $\tilde{g_{2}}: A_{2} \rightarrow A$ and $\tilde{l_{2}}: C_{2} \rightarrow C$ such that . Moreover, $L_{0}=M_{0} \oplus N_{0}$ and $L_{2}=M_{2} \oplus N_{2}$, hence $L_{0}$ and $L_{2}$ are also sums of line bundles of the form $\mathcal{O}_{X}\left(l_{j}\right)$ and $\omega_{X}\left(k_{i}\right)$, respectively. Looking at the cohomology sequence associated to the middle column, it is easy to see that $H_{*}^{1}\left(M_{1}\right)=H_{*}^{1}\left(N_{1}\right)=0$ forces $H_{*}^{1}\left(L_{1}\right)=0$, while $H_{*}^{n-1}\left(M_{1}\right)=H_{*}^{n-1}\left(N_{1}\right)=0$ forces $H_{*}^{n-1}\left(L_{1}\right)=0$.

We check that $\beta_{L} \alpha_{L}=0$; first, notice that any local section $\sigma$ of $L_{0}$ can be written as a sum $g_{1}\left(\sigma_{1}\right)+\tilde{g_{2}}\left(\sigma_{2}\right)$ with $\sigma_{1} \in N_{0}$ and $\sigma_{2} \in M_{0}$. Then

$$
\beta_{L} \alpha_{L}(\sigma)=\beta_{L} \alpha_{L} g_{1}\left(\sigma_{1}\right)+\beta_{L} \alpha_{L} \tilde{g_{2}}\left(\sigma_{2}\right)=l_{1} \beta_{N} \alpha_{N}\left(\sigma_{1}\right)+\tilde{l_{2}} \beta_{M} \alpha_{M}\left(\sigma_{2}\right)=0
$$


since $\beta_{N} \alpha_{N}=\beta_{M} \alpha_{M}=0$. So the middle row is a Horrocks' monad, as desired.

Additionally, one can show that if $X$ be a nonsingular ACM projective scheme of dimension $n \geq 3$ and, then the functor that associates each Horrocks' monad to its cohomology sheaf $E=\operatorname{ker} \beta / \operatorname{im} \alpha$ is additive, exact and full, cf. [12, Theorem 2.6].

4.2. Linear bundles on projective spaces. The following definition is motivated by [10], and it generalizes the concept of mathematical instanton bundle on $\mathbb{P}^{2 m+1}$ introduced by Okonek and Spindler in [15, Definition 1.1].

Definition. A torsion-free coherent sheaf $E$ on $\mathbb{P}^{n}(n \geq 2)$ is called linear if it satisfies the following cohomological conditions:

(1) for $n \geq 2, H^{0}(E(-1))=H^{n}(E(-n))=0$;

(2) for $n \geq 3, H^{1}(E(-2))=H^{n-1}(E(1-n))=0$;

(3) for $n \geq 4, H^{p}(E(k))=0,2 \leq p \leq n-2$ and $\forall k$.

Linear sheaves such that $c_{1}(E)=0$ are called instanton sheaves.

Proposition 10. Linear sheaves form an exact subcategory $\mathcal{L}\left(\mathbb{P}^{n}\right)$ of $\operatorname{Coh}\left(\mathbb{P}^{n}\right)$.

Proof. It is easy to see from the Definition that if $E_{1}$ and $E_{2}$ are linear sheaves, then any sheaf $F$ in the exact sequence

$$
0 \rightarrow E_{1} \rightarrow F \rightarrow E_{2} \rightarrow 0
$$

is also linear, so $\mathcal{L}\left(\mathbb{P}^{n}\right)$ is closed under extensions. Assuming that $F=E_{1} \oplus E_{2}$, we have that $H^{p}(F(k))=H^{p}\left(E_{1}(k)\right) \oplus H^{p}\left(E_{2}(k)\right)$, so closure under direct summands follows easily.

Remark 11. Notice that instanton sheaves form an additive subcategory of $C o h\left(\mathbb{P}^{n}\right)$ which is closed under extensions, but not closed under direct summands.

Several properties of linear torsion-free sheaves on projective spaces are discussed in [10]; see also [11, 12] for properties of linear bundles over more general algebraic varieties.

Recall that a monad on $\mathbb{P}^{n}$ of the form

$$
0 \rightarrow V_{1} \otimes \mathcal{O}_{\mathbb{P}^{n}}(-1) \stackrel{\alpha}{\longrightarrow} V_{2} \otimes \mathcal{O}_{\mathbb{P}^{n}} \stackrel{\beta}{\longrightarrow} V_{3} \otimes \mathcal{O}_{\mathbb{P}^{n}}(1) \rightarrow 0,
$$


where $V_{k}$ are vector spaces, is called a linear monad. Moreover, the cohomology of a linear monad is a torsion-free sheaf if and only if there is a closed subvariety $\Sigma \subset \mathbb{P}^{n}$ of codimension at least two such that the localized map $\alpha(x): V_{1} \rightarrow V_{2}$ is injective for each point $x \in\left(\mathbb{P}^{n} \backslash \Sigma\right)$, see [10, Proposition 4].

The most relevant fact is the following key result relating linear sheaves and linear monads, cf. Proposition 2 and Theorem 3 in [10].

Theorem 12. If $E$ is a linear torsion-free sheaf on $\mathbb{P}^{n}$, then $E$ is isomorphic to the cohomology of the linear monad:

$$
\begin{aligned}
0 & \rightarrow H^{1}\left(E \otimes \Omega_{\mathbb{P}^{n}}^{2}(1)\right) \otimes \mathcal{O}_{\mathbb{P}^{n}}(-1) \rightarrow \\
& \rightarrow H^{1}\left(E \otimes \Omega_{\mathbb{P}^{n}}^{1}\right) \otimes \mathcal{O}_{\mathbb{P}^{n}} \rightarrow H^{1}(E(-1)) \otimes \mathcal{O}_{\mathbb{P}^{n}}(1) \rightarrow 0 .
\end{aligned}
$$

Conversely, the cohomology of a linear monad is a linear sheaf.

We will also need the following result, which is a special case of $[12$, Theorem 2.5]; see also [14, Lemma II.4.1.3].

Lemma 13. If $E$ and $F$ are the cohomology sheaves of two linear monads $\mathcal{V}_{\bullet}(E)$ and $\mathcal{V}_{\bullet}(F)$, respectively, then the map that associates to each homomorphism of monads the corresponding homomorphism of cohomology sheaves is bijective.

Now let us turn our attention to the relation between linear sheaves and twisted representations of the quiver $\left(A_{3}, b a\right)$. Indeed, let $M=$ $H^{0}\left(\mathcal{O}_{\mathbb{P}^{n}}(1)\right)$ and note that the maps $\alpha$ and $\beta$ above can be regarded as matrices of linear polynomials, i.e. $\alpha \in \operatorname{Hom}\left(V_{1}, V_{2}\right) \otimes M$ and $\beta \in$ $\operatorname{Hom}\left(V_{2}, V_{3}\right) \otimes M$. Therefore linear monads are in 1-1 correspondence with twisted representations of the quiver $\left(A_{3}, b a\right)$

$$
V_{1} \underset{\alpha}{\stackrel{M}{\alpha}} V_{2} \underset{\beta}{\stackrel{M}{\beta}} V_{3}
$$

for which the map $\alpha$ is injective and $\beta$ is surjective as maps of sheaves. Such representation will be called admissible; it is easy to see that admissible representations for a full additive subcategory, denoted denoted $\mathcal{A}(n)$, of $\operatorname{Rep}_{M}\left(A_{3}, b a\right)$, the category of all $M$-twisted representations of $\left(A_{3}, b a\right)$. It is also worth noticing that the category $\operatorname{Rep}_{M}\left(A_{3}, b a\right)$ is equivalent to the category of linear representations of a different quiver with relations, providing a version of Theorem 4 for the quiver with relation $\left(A_{3}, b a\right)$. More precisely, consider the 
following quiver, which we denote by $\Gamma_{n}$ :

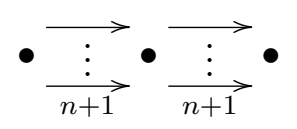

with $\operatorname{dim} M=n+1$ arrows between each vertex. The $n+1$ arrows from the first to the second vertices are denoted $a_{0}, \ldots, a_{n}$, while the $n+1$ arrows from the second to the third vertices are denoted $b_{0}, \ldots, b_{n}$; we impose the following relations:

$$
R_{k, l}: a_{k} b_{l}+a_{l} b_{k}=0, k, l=0, \ldots, n .
$$

Let $\mathbf{R}$ be the set of all relations $R_{k, l}, k \leq l$.

Proposition 14. The equivalence functor $\mathbf{F}: \operatorname{Rep}_{M}\left(A_{3}\right) \rightarrow \operatorname{Rep}\left(\Gamma_{n}\right)$ constructed in the proof of Theorem 4 induces an equivalence of categories $\mathbf{F}: \operatorname{Rep}_{M}\left(A_{3}, b a\right) \rightarrow \operatorname{Rep}\left(\Gamma_{n}, \mathbf{R}\right)$. Moreover, if $V=\left\{\left(V_{1}, V_{2}, V_{3}\right)\right.$, $(\alpha, \beta)\}$ is an admissible $M$-twisted representation of $\left(A_{3}, b a\right)$, then $\mathbf{F}(V)=\left\{\left(V_{1}, V_{2}, V_{3}\right),\left(a_{0}, \ldots, a_{n}, b_{0}, \ldots, b_{n}\right)\right\}$ is such that

(1) there exists $\left(\mu_{0}, \ldots, \mu_{n}\right) \in \mathbb{C}^{n+1}$ such that the map $\mu_{0} a_{0}+\cdots+$ $\mu_{n} a_{n}$ is injective;

(2) the map $\lambda_{0} b_{0}+\cdots+\lambda_{n} b_{n}$ is surjective for every $\left(\lambda_{0}, \ldots, \lambda_{n}\right) \in$ $\mathbb{C}^{n+1}$.

Proof. Choose homogeneous coordinates $\left[x_{0}: \cdots: x_{n}\right]$ in $\mathbb{P}^{n}$; that induces a natural of basis for $M=H^{0}\left(\mathcal{O}_{\mathbb{P}^{n}}(1)\right)$, with respect to which the sheaf maps $\alpha$ and $\beta$ can be written as follows:

$$
\alpha=a_{0} x_{0}+\cdots+a_{n} x_{n} \text { and } \beta=b_{0} x_{0}+\cdots+b_{n} x_{n},
$$

A straightforward calculation shows that $\beta \alpha=0$ if and only if $a_{k} b_{l}+$ $b_{l} a_{k}=0$ for each $k, l=0, \ldots, n$, matching the relations in equation (6). This concludes the proof of the first statement.

Finally, with the above notation in mind, the two conditions in the second part of the Proposition are easily seen to be equivalent to the injectivity of $\alpha$ and the surjectivity of $\beta$.

We are finally in position to prove the main result of this Section.

Theorem 15. $\mathcal{A}(n)$ is an exact subcategory of $\operatorname{Rep}_{M}\left(A_{3}, b a\right)$, which is equivalent to $\mathcal{L}\left(\mathbb{P}^{n}\right)$. 
Proof. The first statement is clear from the definitions. For the second statement, we define a functor:

$$
\mathbf{G}: \mathcal{A}\left(\Gamma_{n}\right) \longrightarrow \mathcal{L}\left(\mathbb{P}^{n}\right)
$$

as follows. Given a $M$-twisted representation $V=\left\{\left(V_{1}, V_{2}, V_{3}\right),(\alpha, \beta)\right\}$ of $\left(A_{3}, b a\right)$, we form the complex of sheaves:

$$
V_{1} \otimes \mathcal{O}_{\mathbb{P}^{n}}(-1) \stackrel{\alpha}{\longrightarrow} V_{2} \otimes \mathcal{O}_{\mathbb{P}^{n}} \stackrel{\beta}{\longrightarrow} V_{3} \otimes \mathcal{O}_{\mathbb{P}^{n}}(1) .
$$

As we mentioned above, $V$ is admissible if and only if the above complex is a linear monad with torsion-free cohomology sheaf (see [10, Proposition 4]), and we define $\mathbf{G}(V)$ as its cohomology sheaf. A morphism between admissible representations will induce a morphism between the corresponding linear monads, which in turn induces a morphism between the corresponding linear sheaves.

Now Theorem 12 implies that the functor $\mathbf{G}$ is essentially surjective, while Lemma 13 implies that $\mathbf{G}$ is fully faithful. This completes the proof.

Theorem 15 allows us to translate geometric properties of sheaves into algebraic properties of the corresponding quiver representations, and vice versa. For example, note that the simple representation of $\left(\Gamma_{n}, \mathbf{R}\right)$ with dimension vector $(0,1,0)$ corresponds, via the above functor, to the trivial line bundle $\mathcal{O}_{\mathbb{P}^{n}}$. The two other simple representations of $\Gamma_{n}$ are not admissible.

Furthermore, given a representation $R$ in $\mathcal{A}\left(\Gamma_{n}\right)$ of dimension vector $\left(v_{1}, v_{2}, v_{3}\right)$, the Chern character of the linear sheaf $\mathbf{F}(R)$ is given by:

$$
\operatorname{ch}(\mathbf{G}(R))=v_{2}-v_{3} \cdot \operatorname{ch}\left(\mathcal{O}_{\mathbb{P}^{n}}(1)\right)-v_{1} \cdot \operatorname{ch}\left(\mathcal{O}_{\mathbb{P}^{n}}(-1)\right) ;
$$

in particular

$$
\operatorname{rk}(\mathbf{G}(R))=v_{2}-v_{3}-v_{1} \text { and } c_{1}(\mathbf{G}(R))=v_{1}-v_{3} .
$$

Therefore, rank $r$ instanton sheaves correspond to representations with dimension vectors of the form $(c, r+2 c, c)$. The integer $c$ is called the charge, of the corresponding instanton sheaf.

It follows from [6, Main Theorem] and the Theorem above that there exists an admissible representation of dimension vector $\left(v_{1}, v_{2}, v_{2}\right)$ if and only if at least one of the following two conditions hold:

- $v_{2} \geq 2 v_{3}+n-1$ and $v_{2} \geq v_{1}+v_{3}$;

- $v_{2} \geq v_{1}+v_{3}+n$. 
Finally, the following interesting statement is an easy consequence of [10, Theorem 22] and Theorem 15 above.

Lemma 16. Every admissible representation $R$ of $\Gamma_{n}$ with dimension vector $(c, n-1+2 c, c)$ where $c \geq 1$ is Schurian, i.e. $\operatorname{Hom}(R, R)=\mathbb{C}$.

As a next step, it would be interesting to study the possible dimension vectors of indecomposable $M$-twisted representations of $\left(A_{3}, b a\right)$ (à la Kac's theorem), and in this way find the possible rank and charge of indecomposable instanton sheaves. We also expect to be able to establish new properties of the moduli spaces of linear sheaves by considering the moduli spaces of the corresponding quivers representations.

\section{References}

[1] H. Abo, and K. Ranestad, Irregular elliptic surfaces of degree 12 in projective fourspace. Math. Nachr. 278, 511-524 (2005).

[2] L. Álvarez-Consúl, O. García-Prada, Dimensional reductions and quiver bundles. J. reine agnew. Math. 556 (2003), 1-46.

[3] W. Barth, K. Hulek, Monads and moduli of vector bundles. Manuscripta Math. 25 (1978), 323-347.

[4] D.-E. Diaconescu, Moduli of ADHM sheaves and local Donaldson-Thomas theory. Preprint math/0801.0820.

[5] M. Casanellas, and R. Hartshorne, Gorenstein biliason and ACM sheaves. J. Algebra 278, 314-341 (2004).

[6] G. Floystad, Monads on projective spaces. Comm. Algebra 28 (2000), $5503-5516$.

[7] S.I. Gelfand, Yu. I. Manin, Methods of homological algebra. Second edition. Springer Monographs in Mathematics. Springer, 1997.

[8] P. B. Gothen, A. D. King, Homological algebra of twisted quiver bundles. J. London Math. Soc. 71 (2005), 85-99.

[9] G. Horrocks, Vector bundles on the punctured spectrum of a local ring. Proc. London Math. Soc. 14 (1964), 689-713.

[10] M. Jardim, Instanton sheaves over complex projective spaces. Collect. Math. 57 (2006), 69-91.

[11] M. Jardim, R. M. Miró-Roig, On the semistability of instanton sheaves over certain projective varieties. Comm. Algebra 36 (2008), 288-298.

[12] M. Jardim, R. Vidal Martins, Linear and Steiner bundles over projective varieties. Comm. Algebra 38 (2010), 2249-2270.

[13] C. Kassel, Quantum groups. Graduate Texts in Mathematics 155. SpringerVerlag, 1995

[14] C. Okonek, M. Schneider, H. Spindler, Vector bundles on complex projective spaces. Boston: Birkhauser (1980).

[15] C. Okonek, H. Spindler, Mathematical instanton bundles on $\mathbb{P}^{2 n+1}$. J. Reine Agnew. Math. 364 (1986), 35-50. 
Representations of quivers on abelian categories and monads on projective varieties $\mathbf{4 2 3}$

[16] B. Szendroi, Sheaves on fibered threefolds and quiver sheaves. Comm. Math. Phys. 278 (2008), 627-641.

São Paulo J.Math.Sci. 4, 3 (2010), 399-423 